\title{
Las Hoyas de la dehesa de Cañal (Salamanca, España), una labor minera antigua de oro secundario
}

\author{
E.J. González-Clavijo(1), J.M. González de Carballo, S. Barrios(2) y E. González-Clavijo ${ }^{(3)}$ \\ (1) Facultad de Ciencias, Universidad de Vigo, Campus Ourense, As Lagoas s/n, E-32004 Ourense, España \\ eduardogclavijo@gmail.com \\ (2) Departamento de Geología, Universidad de Salamanca, Plaza de la Merced s/n, E-37008 Salamanca, España \\ s.barrios@usal.es \\ (3) Instituto Geológico y Minero de España, Unidad de Salamanca, Calle Azafranal 48, ${ }^{\circ}$ A, E-37001 Salamanca, España \\ e.clavijo@igme.es
}

\begin{abstract}
RESUMEN
El paraje conocido como Las Hoyas, en el término municipal de Pelayos (Salamanca), corresponde a una labor minera a cielo abierto de gran antigüedad, en la que se beneficiaba oro sedimentario. Su tipología concuerda con la tradicional en la minería romana, aunque las ruinas existentes en el lugar corresponden a un poblado visigodo. La existencia de varios asentamientos y obras de ingeniería romanas en la comarca, junto con ruinas aún no estudiadas con topónimos de posible origen romano, permiten argumentar que esta explotación minera estuvo activa en el Alto Imperio. Los materiales portadores del oro son continentales de edad cenozoica (Oligocéno) y ocupan una depresión tectónica de origen alpino limitada por fallas de direcciones SO-NE, las principales, y NO-SE, las secundarias. Sus aportes provienen de los cercanos relieves situados al $\mathrm{SE}$, que constituyen las primeras elevaciones del Sistema Central Español.
\end{abstract}

Palabras clave: minería a cielo abierto, minería romana, NO de lberia, oro sedimentario.

\section{Las Hoyas in the "dehesa" of Cañal (Salamanca, Spain), an ancient sedimentary gold mining excavation}

\begin{abstract}
The site known as Las Hoyas, in the municipal area of Pelayos (Salamanca), is a large open-cast mining pit of great antiquity, where sedimentary gold was exploited. Its typology corresponds to that used in traditional Roman mining, but nearby ruins belong to a Visigothic hamlet. The presence of several Roman settlements, roads and bridges in the area, joined to ruins with names of possible Roman origin, not yet studied, suggest the mine was active during the height of the Roman Empire. The sediments bearing the gold are continental of Cenozoic age (Oligocenic) and fill a tectonic groove of Alpine origin confined by SW-NE main faults and NW-SE secondary faults. The source area is the close reliefs placed to the SE, which are the first buttresses of the Spanish Central System.
\end{abstract}

Key words: NW Iberia, open-cast mining, Roman mining, sedimentary gold.

\section{ABRIDGED ENGLISH VERSION}

\section{Introduction and methods}

Evidence of old mining activity is common in the Iberian Peninsula and is frequently of Roman origin. Some of the biggest mines were developed for the recovery of sedimentary gold, an essential raw material for the economy of the Roman Empire. The site known as Las Hoyas (Salamanca province, Spain; Fig. 1) covers a large area (20 ha) displaying a distinct unnatural morphology covered by very old trees (Fig. 2) which suggests a centenary origin for this landscape's anthropic modification. The remains of Roman mines, bridges, potteries and villas are general in the region; but the ruins located in the lower part of Las Hoyas correspond to a Visigothic hamlet. Since the name (Spanish for the hollow) only reflects the shape of the area but it is not 
related to any mining activity, the research was organized into five steps: (1) a preliminary reconnaissance and mapping of the mine works; (2) a detailed review of the geological setting producing a local map; (3) the use of specially calibrated metal-detector devices for the identification of evidence of gold; (4) a study of heavy mineral from a concentrate of sediments; and (5) a bibliographic compilation of Roman remains in the nearby area.

\section{Results and discussion}

Morphologically Las Hoyas consists of an approximately N-S very irregular little valley quarrying the regional plateau (940-960m above sea level in the area). The maximum dip of the excavation is $50 m$ in the eastern half, while the western half reaches only around $30 \mathrm{~m}$ below the plateau surface. The bottom of the hollow presents a number of mining dumps (called murias in Castile) arranged radially from the lower point to facilitate water drainage (Fig. 3). This lower end of the quarry is very close of the E-W flowing Pero Fuertes (Codes) river at the point where an old hamlet was settled. Archaeological excavations in these ruins (EI Cuarto de Las Hoyas Village) estimate $6^{\text {th }}-7^{\text {th }}$ century occupation, thus belonging to the Visigothic epoch in Spain (Storch de Gracia, 1998; Velázquez Soriano, 2000 and 2004; Dahí Elena, 2007). A Roman coin, an as of Tiberius Claudius Nero Drusus, Roman emperor (41-54 AD - height of the Roman Empire), found in the ruins during our research must be considered as not directly related to the settlement, as it presents two drill holes and was presumably later used for ornamental purposes.

The mine is geologically located at the SW corner of the tectonically controlled Douro Meso-Cainozoic Basin, the biggest in the Iberia Peninsula. Tectonic pattern evidence is especially strong in this area, developing a group of SW-NE spurs of the basin bearing a sub-parallel attitude to the best known Ciudad Rodrigo Half-Graben (Fernández Amigot, 1981; Cantano and Molina, 1987). One of these spurs holds the mine and was named the Pero Fuertes Half-Graben -PFHG- in this study (Fig. 4); it was created by a system of alpine faults running along its NW side, which belong to the group called Guijuelo by Martín-Serrano et al. (1991b). Two lithostratigraphic units fill this groove. The lower one consists of white-yellowish conglomerate and arkosic sandstone of estimated Oligocene-Miocene age by lithological correlation to the Ciudad Rodrigo Spur materials (Bellido Mulas et al., 1991), where Oligocene palynomorphs were found (Alonso-Gavilán and Polo, 1986 1987; Polo et al., 1987). The Las Hoyas quarry is located at the upper part of this unit, close to this upper limit to the over-laying conglomerate, sandstone and mudstone red unit of a Miocene-Pliocene age deduced by the stratigraphic position (Bellido-Mulas et al., 1991) and by lithological correlation to other localities of the SW part of the Douro Basin. Both units have been interpreted as a superposition of anastomosing fluvial channels draining areas of arkosic materials, granitic rocks and medium-to high-grade meta-sediments. This interpretation was confirmed by the observed clasts and the obtained heavy minerals, all of them showing provenance from the SE part of the PFHG which constitutes the first elevation of the Alpine age Central Iberian Range. This horst is made of granitic and medium-high metamorphic-grade rocks, while the NW part of the PFHG is the generally high plateau formed only by low-grade meta-sediments.

As no mineral occurrences were found in the cliffs and dumps, the mined material had to be constrained by other means. Some arguments point to a gold mine, since the region presents a number of gold mining works, primary and secondary, of several ages; some of them displaying Roman era evidence of excavation (Junta de Castilla y León, 1985, 1986). In addition, the Pero Fuertes (or Codes) river is a tributary to the medium reach of the Tormes river, where large size gold nuggets have been found (a $63 \mathrm{~g}$ gold nugget from this area is conserved at the Spanish Geological Institute Museum). Nevertheless, other substances (such as tin) may be also the origin of the quarry. A first attempt at discrimination using especially calibrated metaldetector devices was unsuccessful; this suggests the mineralization was formed by very fine grained nuggets.

A new approach was done collecting five samples of sediment in a seasonal water flow draining the quarry (Fig. 3). The samples were concentrated using a pan and then selected by hand under binocular glass (with incorporated camera) in the Geology Department of Salamanca University. All the samples display small (<2 mm) particles of allotriomorphic gold, with a brass yellow colour, characteristic brightness, and an irregular undulated surface (Fig. 5). The rest of the heavy minerals (mainly ilmenite, garnet, and zircon) support the sediment provenance from a medium-high metamorphic-grade area as previously stated. These findings point to a gold recovery for the origin of the big quarry. The shape of the mining work, stopping exactly at the fault which limits the fertile unit, and avoiding the Cambrian meta-sediments, constitutes more data supporting the fact that mine was recovering only sedimentary gold (Fig. 4). A careful reconnaissance of the white quartz dyke intruding this fault did not provide any evidence to support a primary mineralization or mining work related to either the fault or the dyke.

As the Roman recovery method for sedimentary deposits was used during a long period of time after the fall of the empire it is very difficult to ascertain the Roman origin of this gold mine. Nevertheless, a careful bibliographic study was performed to check the Roman era evidence in the area with a positive result. As 
shown in Figure 6 some Roman settlements are known in the environs of Las Hoyas, namely: El Bañadero, Los Casetones II, Anguas, El Cortinal de San Juan, Las Casas del Medrano, and Dehesas Torrecilla I and II. Another group of nearby hamlets belongs to the Visigothic era and some of them could be the continuity of late-Roman villages: El Colmenar, El Cuarto de Remoludo, El Cuarto del Medio, Prado Morán, Los Muladares, Regato Calderón, Prado de los Hoyos, Los Molinos and Salvatierra de Tormes. The Cuarto de las Hoyas Visigothic village, the nearest to the mine, was settled in a position so close the Pero Fuertes river that Roman origin is not plausible; but if it was the processing plant for the mine, the necessity of abundant water justifies the implicit risk of frequent floods. The gold mine and the Visigothic settlement are located by an old important road known as La Calzada de Valverde, which crosses the Tormes river by a Roman bridge (Anguas I) with a funerary monument.

\section{Conclusions}

The big quarry of Las Hoyas de Cañal is a mine for the recovery of sedimentary gold. The mineralization consists of fine gold nuggets disseminated in the Cenozoic detrital sediments which fill an Alpine half-graben located at the SW corner of the Douro Basin. The organization of the dumps inside the quarry preclude the use of controlled erosion as the main mining tool.

The Roman origin has not been properly stated yet, but the abundant manifestations of Roman constructions around the mine strongly suggest that it was started during the Roman Empire and the processing plant was later transformed into a Visigothic hamlet.

\section{Introducción y metodología}

Las evidencias de minas antiguas son abundantes en la península Ibérica y frecuentemente son consideradas de la época romana (Sánchez-Palencia, 1983; Domergue, 1987), dado que la organización del Imperio Romano exigía abundantes recursos minerales de todo tipo para mantener su actividad productiva y su comercio, destacando entre ellos el oro. Las labores mineras romanas para la recuperación de oro secundario en placeres, a veces con movimientos de grandes volúmenes de sedimentos, son bien conocidas en toda Hispania, siendo Las Médulas, en León, su ejemplo más destacado, pero también existen labores mineras atribuidas a los tiempos romanos en otros sectores de la provincia de Salamanca, debiéndose señalar Las Cavenes (Ruíz del Árbol and Sánchez-Palencia, 1999; Sánchez-Palencia and Ruíz del Árbol, 2003; Sánchez-Palencia et al., 2003). Estos hechos generales estimularon nuestra curiosidad sobre el paraje conocido como Las Hoyas de la dehesa de Cañal (Fig. 1), en el término municipal de Pelayos (Salamanca, España). Su gran área con un relieve artificialmente modificado (Fig. 2), que ha dado lugar a su nombre, junto a su evidente antigüedad, puesto que encinas centenarias crecen sobre las escombreras, atrajeron nuestra atención e inspiraron esta investigación sobre el patrimonio minero antiguo.

En esta primera aproximación al yacimiento de Las Hoyas se pretende definir la caracterización arqueológica y geológico-minera de esta labor que ocupa una

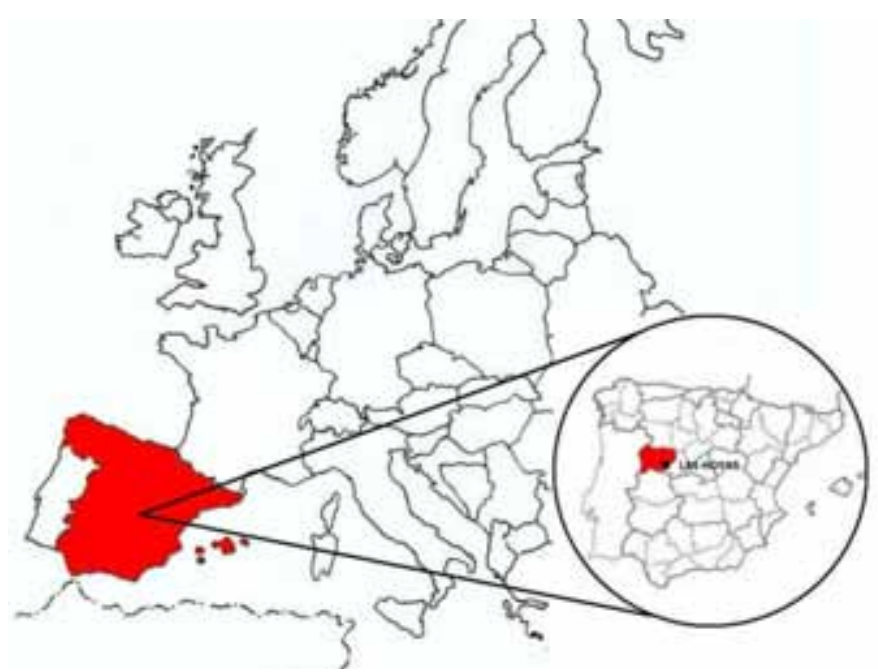

Figura 1. Esquema de situación geográfica de Las Hoyas. Sus coordenadas UTM (WGS 84-Huso 30T) son: 4500000.286285.

Figure 1. Las Hoyas geographical situation sketch. The UTM (WGS 84-30T sector) coordinates are: 4500000.286285.

superficie de 20 ha. Para ello se realizaron varias campañas de campo con diversos objetivos: 1) reconocimiento preliminar y levantamiento del plano de labores; 2) revisión de la geología local sobre la base de los mapas geológicos nacionales de escala 1:50 000 del Instituto Geológico y Minero de España (MAGNA); 3) reconocimiento del área con detectores de metales calibrados para oro; 4) concentración de minerales pesados utilizando bateas de sedimentos recogidos 


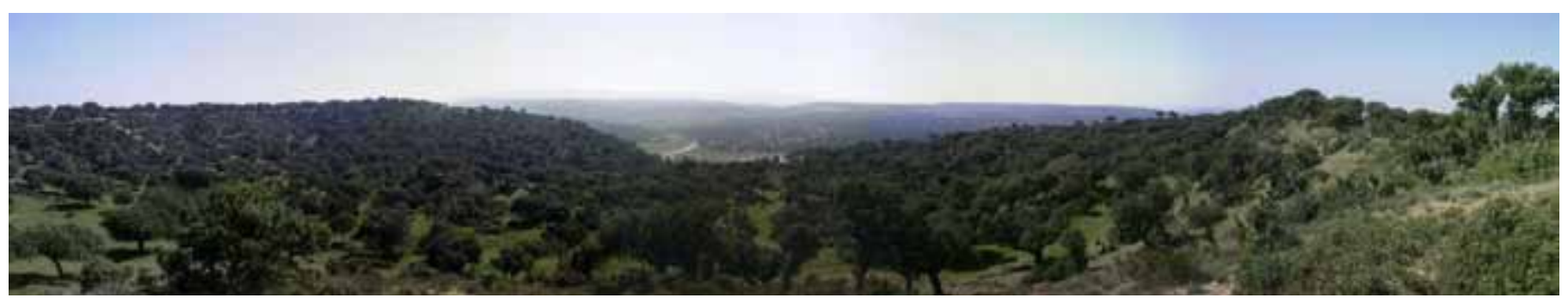

Figura 2. Panorámica hacia el $\mathrm{S}$ de Las Hoyas de Cañal. A pesar de la deformación del montaje fotográfico, se identifica la interrupción de la penillanura, que también está erosionada por el río Codes, visible al brillar su agua en el valle, al fondo, en el centro de la imagen.

Figure 2. Landscape view to the $S$ of Las Hoyas de Cañal. Despite deformation of the photographic composition the quarrying of the plateau is noticeable. In the centre of the image the Codes river valley (bright line of water) naturally erodes the plateau.

dentro de la labor minera; y 5) estudios bibliográficos de los aspectos geológicos y arqueológicos relativos a la zona y al tema de estudio.

\section{Las labores mineras de Las Hoyas}

Cuando a mediados de marzo de 2014 nos propusimos investigar la enorme depresión que con el sugerente nombre de Las Hoyas se localiza en la ladera que domina el poblado visigodo de El Cañal (Fabián et al. 1985,1986; Storch de Gracia, 1998) en la margen derecha del río Codes (o de Pero Fuertes), afluente del Tormes, no imaginábamos la excelente conservación y la perfecta definición de los restos de una mina de oro de tipología romana que albergaba. Afortunadamente han sido muy pocas las transformaciones antrópicas que ha sufrido este yacimiento con el paso del tiempo. Únicamente pequeñas construcciones de uso agropecuario, hoy arruinadas, y cercados para el ganado alteran levemente su superficie. La denominación de Las Hoyas, que evidencia la particular configuración del terreno, indica que el topónimo queda fijado en una época muy posterior a la modificación artificial del espacio, pues solamente se refiere a las características topográficas del lugar, es decir, que ya no había memoria de la explotación minera. En este aspecto contrasta con la toponimia de las anomalías topográficas localizadas en la zona, como la existente cerca de Galinduste (pocos $\mathrm{km}$ al N), en el lugar de Las Minas, que parece acoger restos de estructuras arquitectónicas, muy próximas al punto denominado El Colmenar (Fatás Cabeza et al., 1993), donde aparecieron cerámicas epigráficas y tégulas de los siglos IV-VII dC (Maluquer de Motes, 1957).

Morfológicamente Las Hoyas se nos muestra como una gran vaguada con perímetro irregular, abierta hacia el $\mathrm{S}$ y dispuesta con la suficiente inclinación como para desalojar el agua de escorrentía de la explotación y a su vez facilitar las labores mineras (Fig. 3). No vamos a describir aquí el trabajo en las minas romanas de forma genérica, pues para ello remitimos a los párrafos que los autores antiguos nos dejaron al respecto (básicamente Historia de Diodoro de Sicilia, la Historia Natural por Plinio el Viejo y la conocida Geografía de Estrabón además de escritos de otros autores como Polibio, Estatio o Juan Crisóstomo) y que, grosso modo, pueden dar una idea de algunos de los procedimientos extractivos empleados en el yacimiento. Además desde una perspectiva arqueológica los datos que tenemos de Las Hoyas son pocos y no permiten una descripción ajustada a la realidad. Simplemente recalcar que el método utilizado en esta mina pudo ser semejante al proceso que se desarrolla de forma natural, llamado "de erosión remontante", de manera que origina paisajes muy similares, si bien es cierto que las escombreras localizadas en los bordes superiores de la explotación de Las Hoyas garantizan que se trata de una forma antrópica (Fig. 3).

La mina de Las Hoyas es por lo tanto una explotación a cielo abierto de tipo sedimentario (placeres) en la que se reconocen fácilmente las escombreras ("murias"), producto de la primera selección manual de las piedras más grandes que forman el característico paisaje de majanos que delata los trabajos extractivos. Un estudio superficial de la mina pone en evidencia la existencia de un frente de explotación principal de cerca de $1000 \mathrm{~m}$ de longitud, siendo la diferencia de cota entre las zonas más elevadas y las más bajas de $50 \mathrm{~m}$. Dentro de la concavidad el terreno no se presenta homogéneo pues, si trazamos un eje N-S que divida la mina en dos sectores, tenemos un espacio occidental notablemente más elevado (unos $20 \mathrm{~m}$ ). Las escombreras siguen una disposición groseramente radial con respecto al centro de la explotación y se orientan perpendicularmente en relación a la pendiente del terreno. 


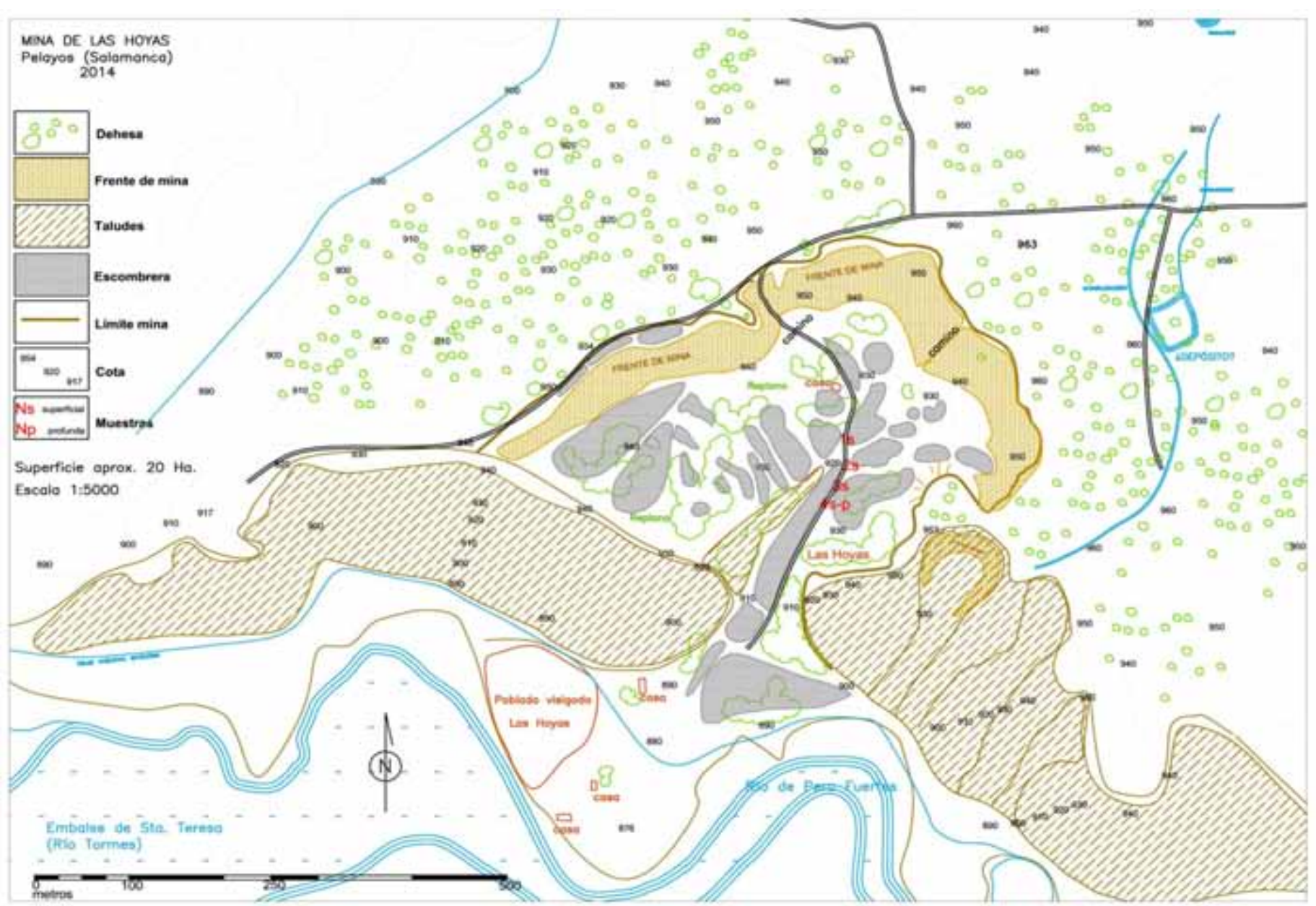

Figura 3. Plano de labores con situación de las muestras tomadas para realizar concentrados por batea (1, 2, 3 y 4 en rojo, siendo s: superficial y p: profunda).

Figure 3. Mining work map showing the sampling spots for concentrates (1, 2, 3 and 4 in red; s: superficial and p: deep).

Utilizando las distintas series históricas de fotografía aérea disponibles podríamos especular (Fig. 3) con la existencia de un canal y un depósito de agua (Sánchez-Palencia and Sastre, 2002) hacia el sector noreste de la explotación (fuera de la mina), que estaría originando un nuevo frente de trabajo, aunque sobre el terreno no ha sido posible confirmarlo en este estudio preliminar. Resulta interesante a nuestro juicio la disposición de las escombreras, ya que su orientación facilita el tránsito sin obstáculos por la mina, evidenciando una cuidadosa planificación a pesar del volumen de los materiales apilados; materiales que conforman los zócalos de bloques de cuarcita y cantos rodados de las construcciones que citan los excavadores del anexo poblado visigodo y que proceden de los ingentes depósitos de rocas acumulados durante la explotación de la mina, que en los siglos VI-VII dC, época estimada de ocupación de la aldea del Cuarto de Las Hoyas (Storch de Gracia,
1998; Dahí Elena, 2007; Velázquez Soriano, 2000; 2004), serían todavía más evidentes que en la actualidad. La presencia de un muro de unos $130 \mathrm{~m}$ de largo y $1,40 \mathrm{~m}$ de ancho que delimita el asentamiento visigodo, cuando menos hacia el E, sobrepasa, a todas luces, la consideración de simple lindero para entrar en la categoría de muralla, aunque la parcial excavación del sitio no permita llegar a conclusiones definitivas en lo relativo a su datación y utilidad. Es necesario comentar que se ha encontrado en estas ruinas una moneda, que por su aspecto y módulo, con el busto mirando hacia la izquierda, es un as del Emperador Tiberius Claudius Nero Drusus, emperador desde el $41 \mathrm{dC}$ al $54 \mathrm{dC}$ (Alto Imperio Romano). Su hallazgo fuera de un contexto arqueológico claro y la presencia de dos orificios en su borde (presumiblemente para ser usada como adorno) nos impide relacionarla cronológicamente y de una forma directa con la explotación minera. 


\section{Contexto geológico general}

El sector de Las Hoyas de la dehesa de Cañal está situado en el extremo SO de la Cuenca del Duero (Fig. 4A). Esta cuenca meso-cenozoica es la de mayor extensión de las existentes en la Península lbérica.
Sus características son intracontinentales y presenta registro sedimentario desde el final del periodo Cretácico hasta el Cuaternario (Alonso-Gavilán et al., 2004). Su control estructural es evidente en casi todos sus bordes y muy intenso en la esquina que nos ocupa. Según Muñoz Martín et al. (2004) este es uno

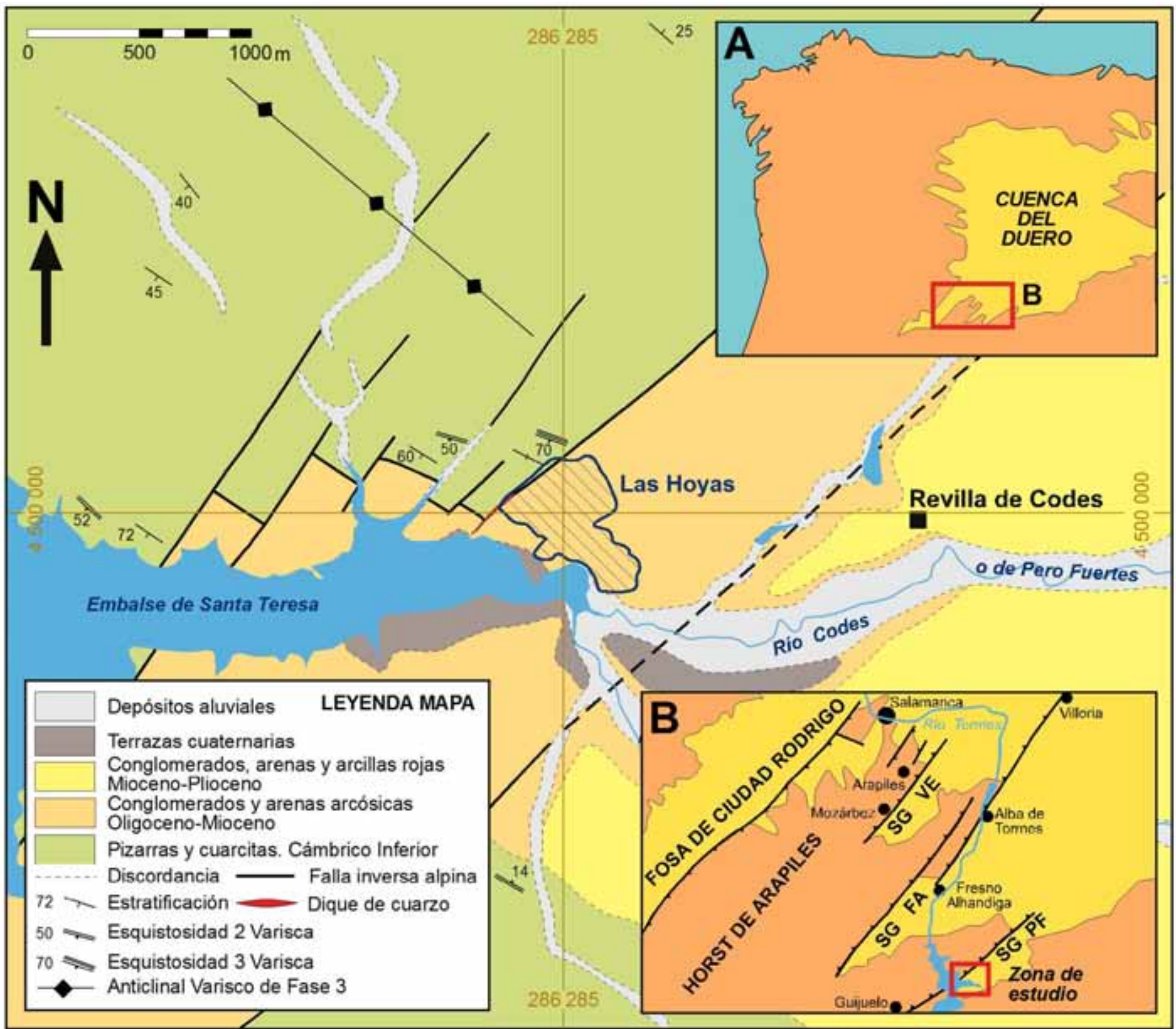

Figura 4. Mapa geológico del sector de Las Hoyas de la dehesa de Cañal con indicación de su situación en la Cuenca Terciaria del Duero (ventana A) y en un esquema estructural simplificado (ventana B) en el que se indican las principales unidades creadas por la tectónica alpina en la parte SO: Fosa de Ciudad Rodrigo, horst de Arapiles, semigraben de Valdescobela (SG VE), semigraben de Fresno Alhándiga (SG FA) y semigraben de Pero Fuertes (SG PF). Coordenadas UTM, WGS84, Huso 30T.

Figure 4. Geological map of the Las Hoyas of the Dehesa of Cañal area showing its situation in the Tertiary Douro Basin (A inset), and a simplified structural sketch (B inset) exhibiting the main units resulting from the alpine tectonics in the SW of the basin: the Ciudad Rodrigo depression, Arapiles horst, Valdescobela half-graben (SG VE), Fresno Alhándiga half-graben (SG FA), and Pero Fuertes half-graben (SG PF). UTM Coordinates, WGS84, 30T Sector. 
de los sectores de lberia donde la cuenca de antepaís alpina se deforma mediante mecanismos de piel gruesa (thickskinned) sin tegumento, formando horsts y grabens, orientados $\mathrm{SO}-\mathrm{NE}$, que definen una serie de espolones (spurs) de materiales de la cuenca que penetran hacia el SO en su basamento relativo, el cual está formado por metasedimentos paleozoicos, rocas ígneas pre-variscas y granitoides relacionados con la orogenia Varisca. El más destacado y conocido de estos espolones es el denominado Fosa de Ciudad Rodrigo (Fig. 4B; Fernández Amigot, 1981; Cantano and Molina, 1987). Estas estructuras son el resultado de la lenta compresión de África y Eurasia durante el Cenozoico (De Vicente et al., 1996; Andeweg et al., 1999; De Vicente et al., 2004) por lo que las fallas que las crean tienen un movimiento relativo entre bloques de tipo inverso, levantando el basamento paleozoico sobre los depósitos cenozoicos, como se observa en varias de ellas a lo largo de la autopista A-66 (Salamanca-Cáceres) cuando cruza esta zona.

Como se muestra en el esquema B de la figura 4, el sector SO de la Cuenca del Duero está dominado estructuralmente por un horst SO-NE que hemos denominado de Arapiles. Hacia el NO está el semigraben complejo que forma la Fosa de Ciudad Rodrigo, mientras que hacia el SE existe un grupo de semigrabens subparalelos a ésta, que hemos denominado: (1) Semigraben de Valdescobela (creado por la falla de Mozárbez); (2) Semigraben de Fresno Alhandiga (generado por el sistema de fallas de AlbaVilloria); y (3) Semigraben de Pero Fuertes (en relación con el sistema de fallas de Guijuelo). En este último espolón, que ya da paso al Sistema Central en su sector de Piedrahita (Ávila), se encuentra la labor minera estudiada. Se han atribuido nuevos nombres a estas tres últimas fosas sedimentarias debido a que el nombre propuesto por Corrochano et al. (1983) integraba todos estos semigrabens en una única unidad estructural denominada depresión de PeñarandaAlba, sin diferenciar en sus mapas las tres cuencas individualizadas que los actuales mapas geológicos reflejan (Martín-Serrano et al., 1991a; Martín-Serrano et al., 1991b; Martín-Serrano et al., 1993; Bellido Mulas et al., 1991).

\section{Geología local de Las Hoyas}

Durante esta investigación se han realizado reconocimientos de campo que han permitido mejorar el detalle de la geología de las hojas MAGNA números 528Guijuelo (Martín-Serrano et al., 1991b) y 529-Santa María del Berrocal (Bellido Mulas et al., 1991), según se refleja en el mapa simplificado de la figura 4. La zona presenta un basamento constituido por metasedimentos de bajo grado metamórfico de edad Cámbrico inferior, pertenecientes a las Formaciones Monterrubio y Aldeatejada (Díez Balda, 1986). Los materiales presentes son, para ambas formaciones, pizarras y limolitas con niveles intercalados de pizarras bandeadas y de materiales detríticos más groseros (conglomerados, areniscas y cuarcitas). Su estructura geológica está generada por la superposición de las diferentes fases de deformación de la orogenia Varisca, siendo la estructura mayor identificable -un anticlinal de dirección NO-SE- de la tercera fase varisca (Díez Balda, 1986; Martín-Serrano et al., 1991b).

La tectónica de edad alpina genera fallas en dos direcciones predominantes, SO-NE las principales, y NO-SE las secundarias, que forman un final geométricamente complejo para el semigraben de Pero Fuertes (Fig. 4). El sistema principal de fallas (SO-NE) es parte del grupo de fallas de Guijuelo (MartínSerrano et al., 1991b) subparalelo a las del Sistema Central que, según Parga (1969), fueron desgarres en dirección que posteriormente se reactivaron en el Mioceno como fallas normales o inversas, siendo esta última cinemática la que se observa en los alrededores de Las Hoyas.

Los depósitos de cobertera que rellenan el semigraben de Pero Fuertes (SGPF) ocupan la parte SE de las fallas del sistema de Guijuelo, habiendo sido descritos y subdivididos de diversos modos, y atribuidos a diversas edades, aunque este último extremo es conflictivo debido a la ausencia de hallazgos fósiles en la región (Martín-Serrano et al., 1991b, Bellido Mulas et al., 1991). Según dichos trabajos se trata de una facies rojas descrita por Bellido Mulas et al. (1991) bajo las casas de la dehesa de Pero Fuertes, unos $3 \mathrm{~km}$ al E de Las Hoyas. Están formadas por arciIlas, arenas y cantos subangulosos de hasta $50 \mathrm{~cm}$ y de diversas litologías, correspondientes a las existentes en los primeros relieves del Sistema Central (sierras de Mercadillo, Narrillos y Ventosa), muy cercanos al SE. Estos depósitos sedimentarios han sido interpretados como un apilamiento de abanicos aluviales proximales con origen en el Sistema Central (AlonsoGavilán et al., 2004). Presentan semejanzas con los descritos en la cuenca del Duero con diferentes nombres y por diferentes autores (Mirazamora, en Corrochano, 1977; Villalba de Adaja, en Corrales, 1982; Conglomerados de Villoria, en Corrochano et al., 1983; Series Rojas, en Martín-Serrano, 1988) y en la Fosa de Ciudad Rodrigo (Rojas del Cubito, en Gracia Plaza et al., 1981; Conglomerados Versicolores, en Jordá, 1983 y Cantano and Molina, 1987; Conglomerado de Cabezuela, en Polo et al., 1987). Su edad y posición 
cronoestratigráfica no están bien determinadas, aunque se considera que esta unidad estratigráfica tiene una edad Mioceno inferior a medio por correlación con la región de Zamora, donde los controles estratigráfico y paleontológico son mejores (Martín-Serrano et al., 1994).

Pero estos materiales rojos se acaban aproximadamente a $1 \mathrm{~km}$ al E de Las Hoyas (Fig. 4), apareciendo bajo ellos unos materiales detríticos de tonos ocre claro a blanquecino, formados por arenas y conglomerados arcósicos, con tamaño máximo de cantos de $40 \mathrm{~cm}$. Las diversas litologías de los cantos (ortogneises, aplitas, pegmatitas, cuarcitas milonitizadas, esquistos biotíticos, etc.) sugieren también una proveniencia de las sierras de Mercadillo, Narrillos y Ventosa, como proponen Alonso-Gavilán et al. (2004). Presentan diversos niveles discontinuos de calcretas, siendo destacable el de $2 \mathrm{~m}$ de espesor que ocupa su techo y da paso a las capas rojas suprayacentes. Este último nivel blanquecino con cemento calcáreo está bien expuesto bajo las casas de la dehesa de Revilla de Codes. Las capas arcósicas son las que han sido explotadas en la mina de Las Hoyas, donde se puede apreciar un espesor mínimo de $80 \mathrm{~m}$, sin que se haya podido observar su base. Por correlación con la Fosa de Ciudad Rodrigo estos materiales se consideran de edad Oligoceno-Mioceno inferior (Bellido Mulas et al., 1991; Martín-Serrano et al., 1993), donde AlonsoGavilán and Polo (1986-1987) y Polo et al. (1987) encontraron palinomorfos fósiles del Oligoceno; si bien trabajos anteriores los consideraban de la base del Mioceno (Pérez González et al., 1979). Se han interpretado como una superposición de canales fluviales individuales trenzados con una carga eminentemente arenosa que drenaban áreas con arcosas, granitos y metasedimentos (Bellido Mulas et al., 1991). Sobre estas dos unidades litoestratigráficas se observan, únicamente, las terrazas y llanuras aluviales de la red fluvial actual.

Morfológicamente la zona está constituida fundamentalmente por una penillanura próxima a la cota de los $1000 \mathrm{~m}$, que en algunos sectores se desarrolla sobre el basamento paleozoico, mientras que en otros está generada sobre la cobertera cenozoica. En los sectores formados por rocas del basamento existen suaves relieves positivos generados por los niveles de sedimentos paleozoicos más resistentes (conglomerados y cuarcitas), mientras que hacia el SE comienzan los relieves escalonados y complejos del borde del Sistema Central español (sierras de Mercadillo, Narrillos y Ventosa). En dicha penillanura se encaja francamente la red fluvial actual, creando laderas más inclinadas en la margen derecha (norte) del río Codes que en su margen izquierda. Las formas claramente artificiales que interrumpen el escarpado talud norte del Codes facilitan la identificación de la enorme labor minera.

Hay que destacar que la labor minera avanzó erosionando los depósitos arcósicos del OligocenoMioceno inferior hasta llegar a la falla que limita la explotación por el NO, en un claro ejemplo del control geológico de los trabajos mineros. Igualmente, el dique de cuarzo que ocupa dicha falla no presenta evidencias de haber sido trabajado, lo que indica que no se trató de un yacimiento mixto primario y secundario de Au.

\section{Estudios minerales}

En este tramo de la cuenca media del ríoTormes (alrededor de Salvatierra de Tormes) es bien conocida la presencia de oro (Mallada, 1896) y, aún en la primera mitad del siglo $X X$, se beneficiaban placeres auríferos mediante bateas, actividad que finalizó al inundar la zona el embalse de Santa Teresa. Más recientemente se conoce la existencia de grandes pepitas de $\mathrm{Au}$, obtenidas junto al embalse y aguas abajo del mismo, durante trabajos de investigación científica (Barrios et al., 2010; Barrios, 2014) mediante el uso sistemático de detectores de metales especialmente calibrados. Es importante destacar que la pepita de Au de mayor tamaño conservada en el Museo Geominero de Madrid (63 g) procede de la zona de Salvatierra de Tormes.

En zonas cercanas, como en las terrazas cuaternarias del río Alhándiga y delTormes, en las zonas de La Maya y Alba de Tormes, hay igualmente referencias de escamas milimétricas de Au y también de grandes pepitas en Calzada de Mendigos (Gil y Maestre, 1880). Más recientemente (Alcalde et al., 1990) se han efectuado prospecciones geoquímicas para diversos elementos en la red de drenaje del área GuijueloCespedosa, concluyendo que el $\mathrm{Au}$ proviene de pequeños filones existentes en los términos municipales de Cabezuela de Salvatierra y Palacios de Salvatierra, habiéndose incorporado a los arroyos de la zona. La existencia de filones de cuarzo aurífero es bien conocida en toda la comarca especialmente en Palacios de Salvatierra (Gil y Maestre, 1880 y Mallada, 1911), pero también en Pizarral de Salvatierra (Franco Herrero et al., 1987; Nespereira et al., 2000), Berrocal de Salvatierra (Junta de Castilla y León, 1985 y 1986) y varias otras zonas de la comarca (Díez Balda and Hacar, 1979).

Aunque el entorno, junto con el tamaño y la tipología de la labor minera, sugerían que se había explotado oro, era necesario confirmar esta presunción. 
Nuestra primera aproximación para determinar la substancia beneficiada en esta mina antigua fue el empleo de detectores de metales. Dicha campaña ofreció un resultado negativo, posiblemente debido a la brevedad de la investigación y, principalmente, al tamaño de las partículas de oro de Las Hoyas, bajo el límite de detección de los aparatos empleados.

Por este motivo se realizó un abordaje diferente y se estudió el concentrado de minerales obtenido tras batear 5 muestras de sedimento superficial recogidas de un arroyo estacional situado en el interior de la labor (Fig. 3). El concentrado fue seleccionado manualmente en el Departamento de Geología de la Universidad de Salamanca, mediante una lupa binocular LEICA (MZ6) con cámara fotográfica integrada (EC) de 3,1 megapixeles.

Todas las muestras bateadas contenían partículas alotriomorfas de Au de tamaño fino (menor de 2 $\mathrm{mm}$ ), color amarillo latón y brillo característico, con superficies irregulares y onduladas. No presentan rasgos cristalinos, ni cuarzo filoniano engarzado. En base al método de Powers (1982), la redondez varía de subangular (Figs. $5 \mathrm{~A}, 5 \mathrm{C}$ y $5 \mathrm{D}$ ) a subredondeada (Fig. 5B) y la esfericidad, de subdiscoidal (Fig. 5A) a subprismoidal (Figs. 5B, 5C y $5 \mathrm{D}$ ).

Dentro de los minerales pesados, además del oro, se han identificado grandes cantidades de ilmenita, y en menor proporción, granate y circón, todos ellos con morfologías desde alotrio a idiomorfas. En menor cantidad, aparecen turmalina, apatito, anatasa y rutilo. Además, se han observado cristales de pirita cúbica limonitizada y algunos cristales de casiterita.

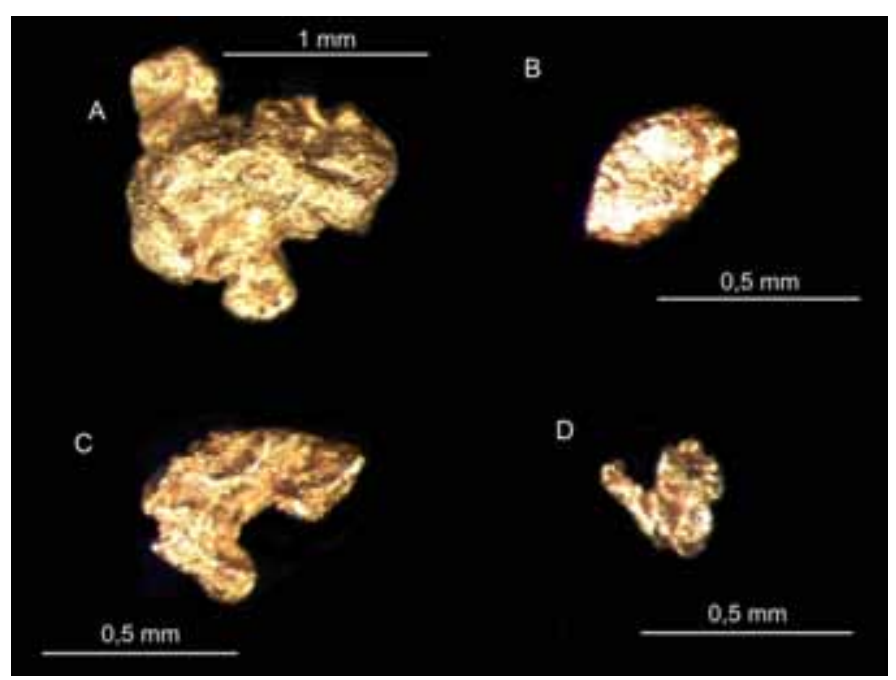

Figura 5. Ejemplos de las pepitas de Au descubiertas en Las Hoyas. A: hallada en la muestra 3S, B: en la 4S, C: en la $1 S$ y D: en la $2 S$. Figure 5. Examples of the gold nuggets discovered at Las Hoyas. A: from sample 3S, B: sample 4S, C: sample $1 S$, and D: sample $2 S$.
Los minerales que forman parte de la fracción ligera corresponden fundamentalmente a cuarzo, feldespato, plagioclasa, moscovita y biotita (cloritizada) con morfología alotrio-hipidiorfa.

El tamaño medio de las partículas del concentrado es generalmente menor de $0.5 \mathrm{~mm}$, aunque en ocasiones pueden alcanzan hasta $3 \mathrm{~mm}$ (pirita, ilmenita, granate o casiterita).

\section{Discusión}

Las formas artificiales de Las Hoyas de la dehesa de Cañal corresponden claramente con las tipologías romanas para beneficiar placeres de Au, pero existía la posibilidad de que la sustancia obtenida fuese otra, estaño por ejemplo, que también es abundante en la zona. La aparición de partículas de $\mathrm{Au}$ a partir de nuestro limitado muestreo (5 bateas) permite asegurar que se trataba de una labor aurífera, dado lo elusiva que es normalmente esta sustancia.

La organización interna de la labor minera, con las escombreras dispuestas para facilitar la circulación por el área de trabajo, nos lleva a descartar la utilización de las técnicas propuestas para otros yacimientos similares, basadas en los derrumbes masivos, más o menos controlados, de los frentes de mina. La meticulosa organización de las escombreras es incompatible con las formas caóticas resultado de las labores extractivas por erosión controlada. Por lo tanto proponemos para la Mina de Las Hoyas un sistema de trabajo manual separando los materiales al mismo tiempo que se avanza en la explotación, desalojando de la excavación únicamente los materiales más finos para su posterior lavado.

Conocer su época de explotación es difícil ya que durante siglos se siguió utilizando la tecnología romana para recuperar minerales pesados de los depósitos sedimentarios. Además, en este aspecto las pruebas son más indirectas, pues no se han encontrado evidencias manifiestamente romanas en el lugar, aunque las explotaciones conocidas en el noroeste ibérico se sitúan cronológicamente entre los siglos I-III dC. Únicamente la moneda romana del siglo I encontrada en las ruinas del poblado visigodo apunta la posibilidad de que se aprovechara para su construcción un emplazamiento anterior. El poblado de Las Hoyas se ubica en el espacio que, por lógica, debería acoger los lugares de lavado de materiales finos de la mina y, por ello, no es arriesgado pensar en la reutilización de las antiguas estructuras mineras abandonadas, no es menos cierto que no parece que haya una continuidad en el uso de un asentamiento rural romano por las poco favorables condiciones de 
habitabilidad que presenta el sitio y que no responden a los habituales estándares de la época (Vitruvio, Los diez libros de arquitectura, Libro I); la presencia muy cercana del río y el lugar propenso a la fuerte erosión de un curso fluvial con grandes variaciones estacionales de caudal, no cumple las mínimas condiciones observadas en la mayoría de los enclaves romanos, con la salvedad precisamente de las instalaciones mineras. Esto último implica la existencia de algún poblado asociado, no necesariamente permanente, pero contemporáneo con la mina, en algún lugar próximo que permita el alojamiento de los trabajadores así como la administración y vigilancia del yacimiento. Hay que tener en cuenta que la cronología para los yacimientos mineros de esta área coincide con el clímax de las explotaciones auríferas en el noroeste de la Hispania romana, es decir entre los siglos I y III dC

Ciñéndonos al entorno más cercano a la mina (Fig. 6) y utilizando los datos conocidos de las prospecciones y excavaciones realizadas (Storch de Gracia, 1998), no hay asentamientos romanos que por su proximidad puedan ser susceptibles de acoger los edificios auxiliares, puesto que el más cercano, temporal y geográficamente, es el de Pero Fuertes (Fabián et al., 1986) clasificado en principio como tardorromano (siglos IV-V dC), siendo los de El Cuarto de En Medio (Storch de Gracia, 1998; Velázquez Soriano, 2004) y EI del Remoludo "aldeas" -según López Quiroga, 2009de época visigoda (siglos VI-VIII). No obstante, un estudio aún superficial del entorno próximo (García Sanjuán, 2005) evidencia la presencia de indicios de estructuras arquitectónicas de adscripción cronológica indeterminada, algunos de ellos por su localización en cerros corresponderían a pequeños espacios fortificados (La Cantera / La Atalaya, Torre Clemente...) (Ariño Gil, 2006; Martín Viso, 2009) pero otros, por su ubicación y estructura abierta, podrían albergar restos romanos (Las Minas, Velayos, Torre Clemente...). Por otra parte, el entorno del embalse de Santa Teresa acoge un elevado número de asentamientos tardorromanos y visigodos (Díaz and Martín Viso, 2011) en los que se podría suponer una continuidad en la ocupación de sitios romanos más antiguos y contemporáneos con el yacimiento minero (Fig. 6).

Así mismo es necesario mencionar la existencia del antiguo camino que con la denominación de Calzada de Valverde de Gonzaliáñez (Morán, 1950), pasaba junto a la mina para después dirigirse siguiendo la vega delTormes a Salvatierra de Tormes, que alberga un asentamiento romano o visigodo y medieval. La denominación calzada suele referirse a caminos medievales o modernos, no obstante, el tra- zado rectilíneo y directo puede corresponder a momentos anteriores. Esta ruta, interrumpida por la construcción del embalse de Santa Teresa, contaba con un puente romano acompañado de un monumento funerario sobre el Tormes, situado en la desembocadura del Codes al Tormes (Maluquer de Motes, 1956; Fatás Cabeza et al., 1993).

Desde el punto de vista de la minería romana conocida en la comarca, un informe de la Junta de Castilla y León $(1985,1986)$ considera que las labores mineras auríferas primarias de El Carrascal y la Dehesa (Palacios de Salvatierra y Berrocal de Salvatierra) presentan evidencias de explotación en tiempos romanos.

Geológicamente los datos son más claros; la unidad geológica fértil es la de conglomerados y arenas arcósicas de edad Oligoceno-Mioceno basal. Además la proximidad en vertical a la secuencia suprayacente de conglomerados, arenas y arcillas rojas sugiere que se explotó la parte alta de la unidad fértil. Ambas unidades rellenan un semigraben de edad alpina con aportes provenientes del cercano Sistema Central, como había sido propuesto por Alonso-Gavilán et al. (2004) y confirmado por nuestras observaciones de las litologías que constituyen los cantos mayores de la secuencia, que se corresponden con las rocas aflorantes al $\mathrm{SE}$, y no con las de bajo grado metamórfico expuestas al NO del semigraben de Pero Fuertes (Bellido et al., 1991). Los minerales pesados identificados en los concentrados de batea (ilmenita, granate, circón, turmalina, apatito, anatasa y rutilo) también sugieren un área fuente con un grado metamórfico medio-alto, como el existente en el sector más cercano del Sistema Central (en concreto la Serie del Álamo y su Complejo Laminar Pegmatoide estudiados por Franco (1974 y 1980) y García de Figuerola et al. (1983).

En definitiva, y aun cuando el hallazgo de una mina de época romana ayuda a completar la visión de la historia antigua del área, no existe por el momento un asentamiento romano contemporáneo al que vincular esta explotación aurífera. Aun así, puesto que habitualmente los asentamientos romanos suelen ubicarse en zonas "seguras", lejos incluso de los lugares afectados por las variaciones más drásticas de los caudales fluviales ("máximas avenidas"), no podemos descartar la presencia de restos en las áreas próximas a la mina y fuera de la zona de afección del embalse de SantaTeresa.

\section{Conclusiones}

Este trabajo presenta, por vez primera, este yaci- 
miento minero antiguo, de gran tamaño y que benefició oro secundario.

Las muestras de oro son de tamaño fino y se encuentran en la unidad estratigráfica inferior del Cenozoico local: los conglomerados y arenas arcósicas de edad Oligoceno-Mioceno inferior. Estos depó-

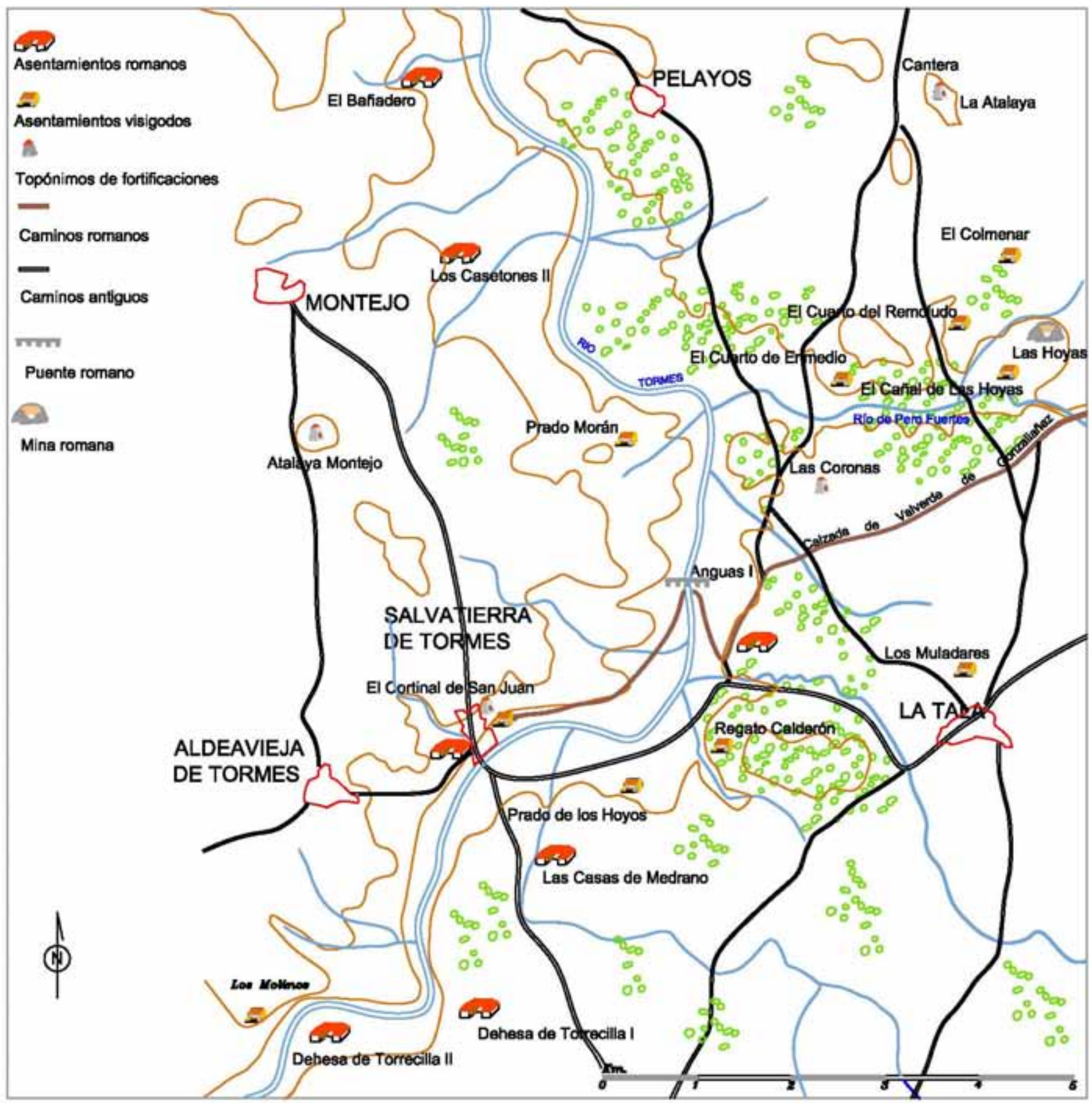

Figura 6. Yacimientos en el entorno del embalse de Santa Teresa. Se han representado los asentamientos romanos, los visigodos, puntos con topología de fortificaciones, un camino romano, un puente romano sobre el río Tormes y la mina estudiada. (Base cartográfica: Mapa Topográfico Nacional, Serie Militar. Hoja 528-Guijuelo. 1947).

Figure 6. Archaeological sites in the area of the Santa Teresa reservoir (Tormes river). Roman and visigothic settlements, places with fortification names, a Roman road, a Roman bridge over the Tormes river and the studied mine are shown. (Cartographic base from: Mapa Topográfico Nacional de España, Serie Militar. Sheet 528-Guijuelo. 1947). 
sitos se encuentran dentro de un semigraben alpino relacionado con el levantamiento del Sistema Central español (semigraben de Pero Fuertes) y en las proximidades de una de las principales fallas inversas que los generan (perteneciente al sistema de fallas de Guijuelo). Los cantos y bloques de esta unidad sedimentaria presentan las litologías deformadas y metamorfizadas que afloran en los cercanos relieves del Sistema Central. Los minerales pesados obtenidos en las 5 bateas estudiadas confirman la proveniencia de un sector con grado metamórfico semejante al de las sierras de Mercadillo, Narrillos y Ventosa.

Se desconoce con detalle el proceso de trabajo en la mina, pero se descarta la utilización de derrumbes masivos en los frentes de mina, a causa de la organización interna observada.

La comarca presenta evidencias de población y minería romana, especialmente al final del imperio. Varios de los establecimientos humanos de la comarca presentan evidencias de continuidad durante la época visigoda.

Por todos estos motivos consideramos que el yacimiento minero aurífero de Las Hoyas del Cañal responde a la constante búsqueda de los minerales necesarios para mantener la compleja estructura económica y administrativa del Imperio Romano, resultando como consecuencia más destacable la alteración de amplias áreas naturales que todavía hoy, casi 2000 años después, muestran el enorme impacto de los trabajos de excavación y procesado de los materiales en las explotaciones.

\section{Agradecimientos}

A Pedro Barba, profesor titular de estratigrafía de la Universidad de Salamanca, por su disponibilidad y las facilidades ofrecidas en el uso de los laboratorios. A Íñigo Orea por la identificación de la moneda romana encontrada. A Ramón González Zapatero (Ramón Valverde) que siempre sostuvo que Las Hoyas de Cañal eran una mina romana de oro y la Calzada de Valverde tenía origen romano, cuyos comentarios nos animaron a emprender esta investigación. La financiación de estos trabajos sólo puede expresarse como en las viejas inscripciones latinas: D.S.P.F. ( $D e$ Sua Pecunia Fecit), dado que han sido realizados sin apoyo o subvención de ninguna clase.

\section{Referencias}

Alcalde, C., Font, X. and Viladevall, M. 1990. Prospección geoquímica a la batea y estudio de las mineralizaciones del área de Guijuelo-Cespedosa (S de Salamanca). Boletín Geológico y Minero de España, 101 (1), 106-112.

Alonso-Gavilán, G. and Polo, M.A. 1986-1987. Evolución tectosedimentaria oligomiocénica del SO de la fosa de Ciudad Rodrigo. Acta Geologica Hispanica. 21-22, 419-426.

Alonso-Gavilán, G., Armenteros, I. Carballeira, A.; Corrochano, A.; Huerta, P. and Rodríguez. J.M. 2004. Cuenca del Duero. In: Vera, J.A. (ed.), Geología de España. SGE-IGME, Madrid, 550-556.

Andeweg, B., De Vicente, G., Cloetingh, S., Giner, J. and Muñoz Martín, A. 1999. Local stress fields and intraplate deformation of Iberia: variations in spatial and temporal interplay of regional stress sources. Tectonophysics, 305: 153-164.

Ariño Gil, E. 2006. Modelos de poblamiento rural en la provincia de Salamanca (España). Entre la Antigüedad y la Alta Edad Media. Zephyrus, 59, 317-337.

Barrios, S. 2014. Estudio del oro sedimentario del SW del Macizo Hespérico Español: rasgos morfo-texturales, geoquímica y geocronología. Tesis Doctoral, Universidad de Salamanca, $415 \mathrm{pp}$.

Barrios, S., Florido, P. and Reguilón, R. 2010. Study of gold nuggets and the regolith located over the SchistGreywacke Complex in the Spanish Central System. SEG Conference, The Challenge of Finding New Mineral Resources, Abstracts Book, 51-54.

Bellido Mulas, F. (dir.), Sánchez Carretero, R., Contreras López, E., Martín Herrero, D. and Klein, E. 1991. Mapa Geológico de España a escala 1:50 000, no 529 Santa María del Berrocal. Instituto Geológico y Minero de España, Madrid, 119 pp.

Cantano, M. and Molina, E. 1987. Aproximación a la evolución morfológica de la "Fosa de Ciudad Rodrigo". Salamanca. España. Boletín de la Real Sociedad Española de Historia Natural (Geología), 82 (1-4), 87-101.

Corrales, I. 1982. El Mioceno al sur del Río Duero, Sector Occidental. Temas Geología y Minería. 6 (2). 709-713.

Corrochano, A. 1977. Estructura y sedimentología del Paleógeno de la provincia de Zamora. Tesis doctoral, Universidad de Salamanca, $336 \mathrm{pp}$.

Corrochano, A., Carballeira, J., Pol, C. and Corrales, I. 1983. Los sistemas deposicionales terciarios de la depresión de Peñaranda-Alba y sus relaciones con la fracturación. Stvdia Geológica Salmanticensia, XIX, 187-199.

Dahí Elena, S. 2007. Un contexto cerámico de la Antigüedad Tardía: el yacimiento de San Pelayo. Nuevos datos sobre la cronología de las pizarras visigodas. Pirenae, 38 (I), 94-95.

De Vicente, G., Giner, J.L., Muñoz-Martín, A., GonzálezCasado, J.M. and Lindo, R. 1996. Determination of present-day tensor and neotectonic interval in the Spanish Central System and Madrid Basin, central Spain. Tectonophysics, 266, 405-424.

De Vicente, G., Vegas, R., Guimerà, J., Muñoz-Martín, A., Casas, A., Martín Velázquez, S., Heredia, N., RodríguezFernández, L.R., González-Casado, J.M., Cloetingh, S., Andeweg, B., Álvarez, J. and Olaiz, A. 2004. Evolución geodinámica cenozoica de la plaza ibérica y su registro en el antepaís. In: Vera, J.A. (ed.), Geología de España. SGE-IGME, Madrid, 597-602. 
Díaz, C.P. and Martín Viso, I. 2011. Between taxation and rent. Fiscal problems from Late Antiquity to Early Ages. (Separata). Edipuglia Bari, 253 pp.

Díez Balda, M.A. and Hacar, M. 1979. Investigación geológico-minera de la zona de Guijuelo (Salamanca). Vol. I: Estudio geológico de la Hoja de Guijuelo. Memoria y Mapa, IGME, Div. Minería, 81 pp.

Díez Balda, M.A. 1986. El Complejo Esquisto-grauváquico, las series paleozoicas y la estructura hercínica al sur de Salamanca. Acta Salmanticensia, Ivssv Senatvs Vniversitatis Edita, Ciencias, 52, 162 p.

Domergue, C. 1987. Catalogue des mines et des fonderies antiques de la Péninsule Ibérique, Publications de la Casa de Velázquez, Série Archéologie VIII, Madrid. 713 pp.

Fabián, J.F., Santonja, M., Fernández, A and Benet, N. 1986. Los poblados hispano-visigodos de Cañal, Pelayos (Salamanca). Consideraciones sobre el poblamiento entre los siglos $\mathrm{V}$ y VIII en el S.E. de la provincia de Salamanca. Estudios Arqueológicos, I, 9-25.

Fatás Cabeza, G., Caballero Zoreda, L., García Merino, C and Cepas Palanca, A. (eds.) 1993. Hoja K-30: Madrid, Cæsaravgvsta - Clvnia, Tabvla Imperii Romanii (Unión Académica Internacional), Instituto Geográfico Nacional, Madrid, 339 pp.

Fernández Amigot, J.A. 1981. Prospección e investigación de yacimientos uraníferos en la provincia de Salamanca. Tecniterræ, 43, 45-73.

Franco González, M.P. 1974. Las formaciones infraordovícicas y el borde de las granodioritas al Este de Guijuelo (Salamanca). Tesis de Licenciatura, Universidad de Salamanca, $38 \mathrm{pp}$.

Franco González, M.P. 1980. Estudio petrológico de las formaciones metamórficas y plutónicas al Norte de la depresión del Corneja-Amblés (Sierra de Ávila). Tesis Doctoral, Universidad de Salamanca, 273 pp.

Franco Herrero, A., García Sánchez, A., Gonzalo, F. and Gracia, A. 1987. Caracteres geoquímicos y mineralógicos del yacimiento de $\mathrm{Sn}$ y $\mathrm{Au}$ de Montejo (Salamanca). Cuaderno do Laboratorio Xeolóxico de Laxe, 11, 349356.

García de Figuerola, L.C., Franco González, P. and Castro Dorado, A. 1983. Características petrológicas del Complejo laminar Pegmatoide (Serie del Álamo) de las provincias de Salamanca y Ávila. Stvdia Geologica Salmanticensia, XIX, 33-77.

García Sanjuán, L. 2005. Introducción al reconocimiento y análisis arqueológico del territorio. Ariel Prehistoria. Barcelona. 32-57.

Gil y Maestre, A. 1880. Descripción física, geológica y minera de la provincia de Salamanca. Comisión del Mapa Geológico de España, Madrid, 299 pp.

Gracia Plaza, A.S., García Marcos, J.M. and Jiménez, E. 1981. Las fallas de "El Cubito": Geometría, funcionamiento y sus implicaciones cronoestratigráficas en el Terciario de Salamanca. Boletín Geológico y Minero, 92 (6), 267-273.

Jordá Pardo, J.F. 1983. Evolución morfogenética de la vertiente NW de la Sierra de Francia y su relación con la
Fosa de Ciudad Rodrigo. Stvdia Geologica Salmanticensia, 8, 129-168.

Junta de Castilla y León 1985. Estudio de posibilidades de minería de oro en la Comunidad Autónoma de Castilla y León. Fondo documental de la Dirección General de Minas de la Junta de Castilla y León (documento inédito).

Junta de Castilla y León 1986. Investigación de la Reserva del Estado Salamanca-1. Fondo documental de la Dirección General de Minas de la Junta de Castilla y León (documento inédito).

López Quiroga, J. 2009. Arqueología del hábitat rural de la Península Ibérica (siglos V-X). La Ergástula, Madrid, 101102.

Mallada, L. 1896. Explicación del Mapa Geológico de España. Tomo II. Sistemas Cambriano y Siluriano. Memorias de la Comisión del Mapa Geológico de España, 20, 516 pp.

Mallada, L. 1911. Explicación del Mapa Geológico de España. Tomo VII. Sistemas plioceno, diluvial y aluvial. Memorias de la Comisión del Mapa Geológico de España, 25, 543 pp.

Maluquer de Motes, J. 1956. La escultura visigoda de Salvatierra de Tormes, Zephyrus, VII, 87-91.

Maluquer de Motes, J. 1957. Carta Arqueológica de España, Provincia de Salamanca. Salamanca. 158 pp.

Martín-Serrano, A. and Monteserín López, V. (dir.), Díez Balda, M.A., Bellido Mulas, F., García Casquero, J.L., Santiesteban Navarro, J.L. 1991a. Mapa Geológico de España a escala 1:50 000, $n^{\circ} 503$ Las Veguillas. Instituto Geológico y Minero de España, Madrid, 92 pp.

Martín-Serrano, A. and Monteserín López, V. (dir.), Mediavilla López, R., Bellido Mulas, F., Díez Balda, M.A., García Casquero, J.L. and Robles Casas, R. 1991b. Mapa Geológico de España a escala 1:50 000, nº 528 Guijuelo, Instituto Geológico y Minero de España, Madrid, 109 pp.

Martín-Serrano, A. and Monteserín López, V. (dir.), Santiesteban Navarro, J.L.;,Díez Balda, M.A. and Nozal Martín, F. 1993. Mapa Geológico de España a escala 1:50 000, $n^{\circ} 478$ Salamanca, Instituto Geológico y Minero de España, Madrid, 96 pp.

Martín-Serrano, A. and Monteserín, V. (dir.), Villar Alonso, P., Díez Montes, A., Santiesteban Navarro, J.I., Carral González, P. and Rubio Pascual, P. 1994. Mapa Geológico de España a escala 1:50 000, $n^{\circ} 477$ Barbadillo, Instituto Geológico y Minero de España, Madrid, 101 pp.

Martín-Serrano, A., 1988. El relieve de la región occidental zamorana. La evolución geomorfológica de un borde del macizo Hespérico. Instituto de Estudios Zamoranos Florián Docampo, Diputación de Zamora, Zamora, 306 pp.

Martín Viso, I. (ed.) 2009. ¿Tiempos oscuros? Territorios y sociedad en el centro de la Península lbérica (siglos VIIX). Silex, Madrid, 93-106.

Morán, C. 1950. Antiguas vías de comunicación en Salamanca. Revista de Obras, 98-I (2828). Ministerio de Fomento, $607 \mathrm{pp}$.

Muñoz Martín, A. (coord.), Álvarez, J., Carbó, A., De Vicente, G., Vegas, R. and Cloetingh, S. 2004. La estructura de la corteza de antepaís ibérico. In: Vera, J.A. (ed.), Geología de España SGE-IGME, Madrid, 592-597. 
Nespereira, J., Moro, M.C., Crespo, J.L., Villar, P. and Fadón, O. 2000. Aportaciones al conocimiento geológico y mineralógico del yacimiento de Sn-Au de Mina Dominica (Salamanca). Geotemas, 1 (4), 57-62.

Parga, J.R. (1969). Sistemas de fracturas tardihercínicas del Macizo Hespérico. Trabajos del Labotario Xeolóxico de Laxe, 37, 1-15.

Pérez González, A., Elizaga, E. (coord.), Vargas, I, Peón, A., García de Figuerola, L.C. y Franco, P. 1979. Mapa Geológico de España a escala 1:50 000, no 504 Alba de Tormes. Instituto Geológico y Minero de España, Madrid.

Polo, M.A., Alonso Gavilán, G. and Valle, F.M. 1987. Bioestratigrafía y paleobiografía del Oligoceno-Mioceno del borde SO de la fosa de Ciudad Rodrigo (Salamanca). Stvdia Geologica Salmanticensia, 24, 229-245.

Powers, 1982. Comparison chart for estimating roundness and sphericity. American Geological Institute, 18, 1-2.

Ruíz del Árbol, M. and Sánchez-Palencia, F.J. 1999. La minería aurífera romana en el noroeste de Lusitania: Las Cávenes de El Cabaco (Salamanca). Archivo Español de Arqueología, Madrid, 72, 119-139.

Sánchez-Palencia, F.J. 1983. La explotación del oro de Asturias y Gallaecia en la antigüedad. Tesis Doctoral, Universidad Complutense, Madrid.
Sánchez-Palencia, F.J. and Ruíz del Árbol, M. 2003. La zone minère de Las Cávenes de El Cabaco et du PinalejoTenebrilla (Salamanque). Atlas Historique des zones minières $d^{\prime}$ Europe (A. Orejas ed.), Vol. II, $12 \mathrm{pp}$.

Sánchez-Palencia, F.J. and Sastre, I. 2002. La red hidráulica en las minas romanas de oro del noroeste hispano. Artifex Ingeniería Romana en España. Museo Arqueológico Nacional. Catálogo de Exposición, Madrid, 251-254.

Sánchez-Palencia, F.J. and Ruíz del Árbol, M., López, O. and Moreno, E. 2003. Tierra, Agua y Oro. Arqueología del Paisaje en la Sierra de Francia. Junta de Castilla y León, Consejería de Cultura y Turismo, 79 pp.

Storch de Gracia, J.J. 1998. Avance de las primeras actividades arqueológicas en los yacimientos hispano-visigodos de la Dehesa del Cañal (Pelayos, Salamanca). Los visigodos y su mundo, Madrid, 141-161.

Velázquez Soriano, I. 2000. Documentos de época visigoda escritos en pizarra (siglos VI-VIII). Monumenta Palaeographica Medii Aevi, Series Hispanica. Turnhout, 15, 31-32.

Velázquez Soriano, I. 2004. Las pizarras visigodas (Entre el latín y su disgregación. La lengua hablada en Hispania, siglos VI-VIII). Instituto Castellano y Leonés de la Lengua, Burgos, 188-190.

Recibido: enero 2015

Revisado: mayo 2015

Aceptado: junio 2015

Publicado: marzo 2018 